\title{
Constraining the Intergalactic Medium Enrichment History with QSO Pairs
}

\author{
Evan Scannapieco ${ }^{1}$ and Crystal L. Martin ${ }^{2}$ \\ ${ }^{1}$ School of Earth and Space Exploration, Arizona State University, P.O. Box 871404, Tempe, \\ AZ, 85287-1404 \\ email: evan.scannapieco@asu.edu \\ ${ }^{2}$ Dept. of Physics, University of California, Santa Barbara, CA, 93106
}

\begin{abstract}
Intergalactic metals are ubiquitous, but their sources remain unknown. A key constraint on these sources is the spatial distributions of metals. Yet, the clustering of metals is difficult to interpret along single lines-of-sight, because distance and velocity information are mixed in redshift space. To overcome this situation we are carrying out detailed comparisons between the line-of-sight and transverse distributions of metal line absorption systems observed in a large sample of QSO pairs and simulations including a wide range of IGM-enrichment scenarios. The degeneracy between distance and velocity is broken by the transverse information available in pairs of sightlines, and thus these comparisons are providing unique new constraints on when and where metals were ejected from galaxies.
\end{abstract}

Keywords. intergalactic medium, QSO absorption lines

\section{Introduction}

The intergalactic medium (IGM) spans the most remote and empty regions of the universe. However, even this most rarified of environments is teeming with the byproducts of stellar evolution. In fact, for over 15 years, quasar (QSO) absorption-line studies have shown that the IGM is filled with heavy elements (e.g. Tytler et al. 1995; Songaila \& Cowie 1996), which are highly inhomogeneous (Rauch, Haehnelt \& Steinmetz 1997), and present even at high redshifts (Becker et al 2009; Ryan-Weber et al. 2009).

Yet despite these many observations, the sources of intergalactic metals remain unknown. There were numerous starburst-driven outflows at $z \approx 3$ (e.g. Pettini et al. 2001) but the evidence pointing toward them being the driving force behind the majority of enrichment is unclear. Instead, there are strong theoretical arguments that suggest that these galaxies may only represent the tail end of a larger number of smaller starbursts at higher redshifts (Scannapieco, Ferrara, \& Madau 2002) that may have been much more important for IGM enrichment. Indeed, a number of studies suggest that the highestredshift metal-free stars may have been extremely massive, resulting in a large number of supernovae that would have distributed metals at redshifts of $z \geqslant 15$. (e.g. Bromm et al. 2001; Schneider et al.2002; Abel et al. 2002; Heger \& Woosley 2002).

Regardless of which objects enriched the IGM, it is clear that they must have formed in dense regions of space, which are far more clustered than the overall matter distribution. This "geometrical-biasing," is a systematic function of the masses of these structures (Kaiser 1984). Thus, information from the large scale clustering of metal absorbers provides valuable insight into the objects from which metals were ejected.

Scannapieco et al. (2006) studied this spatial distribution in high-resolution, high signal-to-noise VLT (UVES) spectra of absorbers at redshifts $z=2-3$. They computed a line-of-sight correlation function in redshift space, and found that it had a high 
amplitude and a 'knee' at $\approx 150 \mathrm{~km} / \mathrm{s}$. By comparing this with a suite of simulations, they found that these measurements were consistent with bubbles of metals $\approx 2$ comoving $\mathrm{Mpc}$ in radius, surrounding dark matter halos with masses $\approx 10^{12} M_{\odot}$. However, the clustering of metals is difficult to interpret along single lines of sight, because distance and velocity information are mixed in redshift space.

\section{Method}

To overcome this degeneracy we made use of a sample of 29 quasar pairs, with redshifts between 1.7-4.3 and separations from $\approx 0.1$ to 2 comoving Mpc (Martin et al. 2009), which were selected from the ongoing work of Hennawi et al. (2006,2009). Spectra for each pair were taken with the ESI spectrograph on Keck II, which provided a resolution of $60 \mathrm{~km} \mathrm{~s}^{-1}$, and continuous coverage from $4,000 \AA$ to $10,000 \AA$. For this study we focused on CIV absorbers, whose 1548.204, 1550.781 doublet is the best tracer of metals in highly-ionized intergalactic gas.

The absorbers were identified by inspection, fitted by a pair of Gaussian line profiles if they were well separated from other absorption lines, and fitted using the SPECFIT program (Kriss 1994) in the case of blends. For most spectra, this allowed us to detect CIV absorbers at the $5 \sigma$ level down to (1550.781) equivalent widths of $0.02 \AA$. Our full data set included 450 absorbers, but as we are interested in the general distribution of intergalactic metals, we flagged absorbers within $5,000 \mathrm{~km} \mathrm{~s}^{-1}$ of the QSO as associated absorbers and excluded them from the analysis. This left a sample of 316 intervening CIV absorbers, with a median redshift of 3.0 .

To compare with this data set, we used the Gadget-2 code (Springel 2005) to perform a large numerical simulation that contained $700^{3}$ dark matter particles, each with a mass of $3.35 \times 10^{7} M_{\odot}$, in a periodic cubic region of the universe, 50 comoving $\mathrm{Mpc} / \mathrm{h}$ on a side. Initial conditions were generated using the Grafic code (Berschinger 2001), and evolved to $z=1.7$ assuming a $\Lambda$ CDM model with a Hubble constant of $70 \mathrm{~km} / \mathrm{s}$, and $\Omega_{0}=0.3$, $\Omega_{\Lambda}=0.7, \Omega_{b}=0.046$, and $\sigma_{8}=0.9$, where $\Omega_{0}, \Omega_{\Lambda}$, and $\Omega_{b}$ are the total matter, vacuum, and baryonic densities in units of the critical density, and $\sigma_{8}^{2}$ is the variance of linear fluctuations on the $8 h^{-1} \mathrm{Mpc}$ scale.

Outputs of the simulation were written once per light crossing time of the box, and for each output we identified bound dark-matter groups with masses above $10^{10} M_{\odot}$ using a friends-of-friend algorithm (Davis et al. 1985). Following the approach in Pichon et al. (2003), we selected all groups above a threshold group mass $M_{s}$ as markers of the centers of enrichment events, about which we painted spheres of radius $R_{s}$ which we took to represent regions containing metals. All smaller groups within these regions were associated with CIV absorbers, leading to modeled metal distributions as ilustated in Figure 1, which shows the results of two choices of of $R_{S}$ with $M_{s}$ fixed at $5 \times 10^{11} M_{\odot}$

For each pair of quasars we cast two sightlines through the simulation outputs, separated by the appropriate angular separation and covering the appropriate redshift range. Each time a sightline passed near one of the groups associated with CIV absorbers, we added an absorption line system to our sample, with an equivalent width which was set by the impact parameter between the sightline and the absorber, such that the overall column density distribution was the same as in observations. Finally, for each simulated sightline pair, we imposed the same noise limits as in the real data, giving us simulated data sets with the same properties as the observed data. 


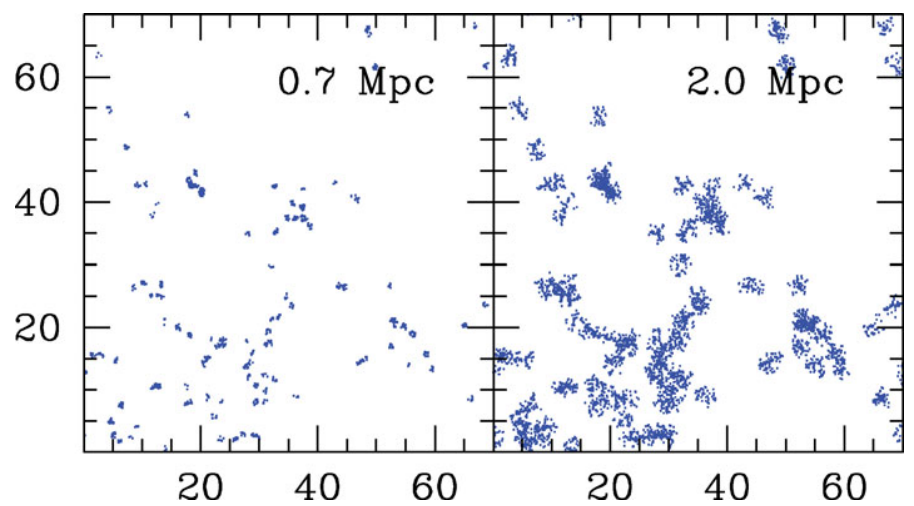

Figure 1. Distribution of intergalactic metals at $z=2.9$ in two simulated models. In the left panel, metal-line systems are relatively near (within 0.7 comoving Mpc) of $5 \times 10^{11} M_{\odot}$ halos In the right panel, metal-line systems are clustered at relatively large distances from $5 \times 10^{11} M_{\odot}$ halos (up to 2.5 comoving Mpc), as would be expected if most intergalactic metal enrichment took place during the epoch of reionization.

\section{Results}

With these models in hand, we were able to directly compare our simulated measurements of both the line-of-sight correlation function and transverse correlation function. Along the line-of-sight, this was computed by counting up pairs as a function separation, and dividing by the number of pairs expected for a random spatial distribution of absorbers, subjected to the same noise limits as in the observations. For the transverse correlation funciton, this was computed by matching up all pairs of absorbers within $1,000 \mathrm{~km} \mathrm{~s}^{-1}$ of each other, and again dividing by the number of such pairs in a random spatial distribution of absorbers subjected to the same noise limits as in the data.

While this analysis is still in progress, Figure 2 shows the results of a comparison between the observations a model with $M_{s}=5 \times 10^{11} M_{\odot}$ and $R_{s}=2.5 \mathrm{Mpc}$. In general, this large bubble model, which is similar to the one fit in Scannapieco et al. (2006) provides a good match to both the line-of-sight and transverse correlation functions. On the other hand, decreasing the bubble size, $R_{s}$ or changing the mass of the central dark matter halos, $M_{s}$, leads to a poorer fit to both the line-of-sight and transverse correlation functions simultaneously.

Note this large bubble size is more easily accommodated as arising from the overlap of numerous clustered sources at very high-redshifts (e.g. Scannpieco 2005) than it is from single bubbles driven by outflows from $z \approx 3$ Lyman break galaxies. Indeed, for winds to reach these large distances within the $\approx 100-1,000$ Myr timescale over which a galaxy is most likely to be identified by the drop-out technique (e.g. Ferguson et al. 2002; Shapley et al. 2005) would require typical velocities between 1,000 and $10,000 \mathrm{~km} / \mathrm{s}$, which are not realistic.

Furthermore, we considered a simulation model with ongoing winds, in which we applied a boost of velocity $v_{\text {boost }}$ pointed radially outward from the large $M_{s}$ halos to each of lower halos associated with CIV absorbers. In general introducing this additional parameter did not improve the fits between the models and the simulations, suggesting that widespread outflows at $z \leqslant 4$ may not play a role in determining the spatial and velocity distribution of most CIV systems. Further observations and simulations are ongoing, which are quickly closing in on the sources of intergalactic metals. 

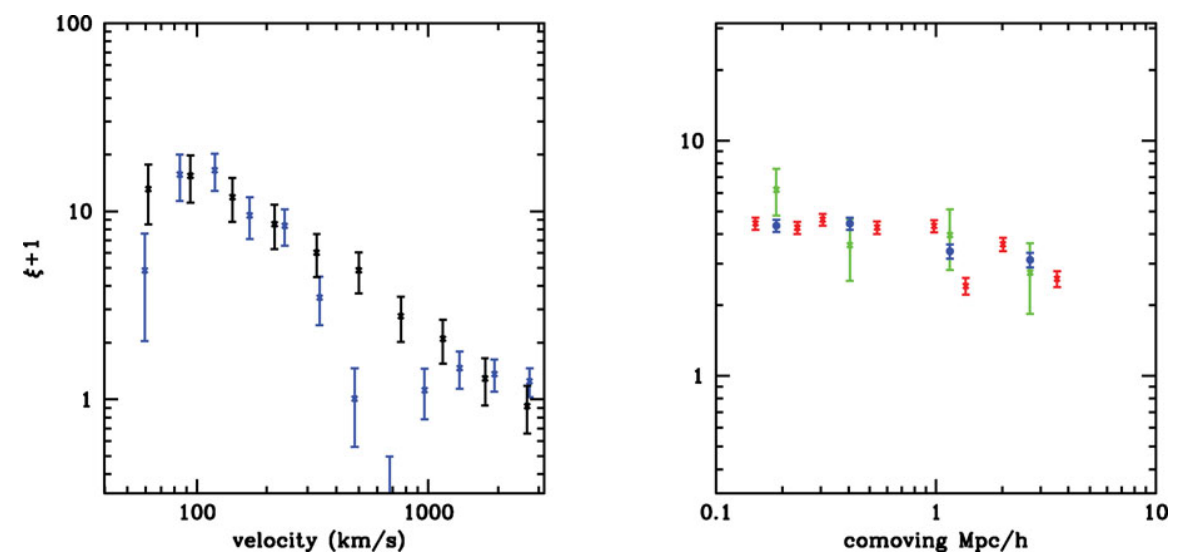

Figure 2. Line-of-sight correlation function for our observed data set (blue points) and simulations (black points) with $M_{s}=5 \times 10^{11} M_{\odot}$ and $R_{s}=2.5 \mathrm{Mpc}$. Transverse correlation function from the observations divided up into 8 bins (red points) and 4 bins (blue points) with an equal number of expected pairs, as compared to the transverse correlation function derived from the simulations (green points) with $M_{s}=5 \times 10^{11} M_{\odot}$ and $R_{s}=2.5 \mathrm{Mpc}$.

\section{References}

Abel, T., Bryan, G. L., \& Norman, M. L. 2002, Science, 295, 93

Becker, G. D., Rauch, M., \& Sargent, W. L. W. 2009, ApJ, 698, 1010

Bertschinger E. 2001, ApJS, 137, 1

Bromm V., Ferrara A., Coppi P. S., \& Larson R. B. 2001, MNRAS, 328, 969

Cowie, L. L., Songaila, A., Kim T.-S., \& Hu, E. M. 1995, AJ, 109, 1522

Davis, M., Efstathiou, G., Frenk, C. S., \& White, S. D. M. 1985, ApJ, 292, 371

Feguson, H. C., Dickenson, M., \& Papovich, C. 2002, ApJ, 569, 65

Heger, A. \& Woosley, S. E. 2002, ApJ, 567, 532

Hennawi, J. F. et al. 2006, AJ, 131, 1

Hennawi, J. F. et al. 2009, ApJ, submitted (arXiv:09083907)

Kaiser N. 1984, ApJ, 284, L9

Kriss, G. 1994, A. S. P. Conference Series, 61, 437

Pettini M. et al. 2001, ApJ, 554, 981

Pichon, C. et al. 2003, ApJL, 597, 97

Martin, C. L. et al. 2009, in preperation

Rauch M., Haehnelt M. G., \& Steinmetz M. 1997, ApJ, 481, 601

Rauch M., Haehnelt M. G., \& Steinmetz M. 1997, ApJ, 481, 601

Scannapieco E., Ferrara A., \& Madau P. 2002, ApJ, 574, 590

Scannapieco E. 2005, ApJL, 724, 1

Scannapieco E., et al. 2006, MNRAS, 365, 615

Schneider R., Ferrara A., Natarajan P., \& Omukai K. 2002, ApJ, 571, 30

Shapley, A. E. et al. 2005, ApJ, 626, 698

Springel V. 2005, MNRAS, 364, 1105

Tytler, D., et al. 1995 in QSO Absorption LInes, ed. G. Meylan, 289 